\title{
Accuracy of heartbeat perception is reflected in the amplitude of the heartbeat-evoked brain potential
}

\author{
OLGA POLLATOS AND RAINER SCHANDRY \\ Biological Psychology, Department of Psychology, Ludwig-Maximilians-University Munich, Germany
}

\begin{abstract}
Neurotransmission from the heart to the brain results in a heartbeat-evoked potential (HEP). In this study, the influence of the ability to detect one's heartbeats based on the HEP was examined. According to their results in a heartbeat perception task, subjects were classified as good $(n=18)$ or poor $(n=26)$ heartbeat perceivers. EEG, EOG, and ECG were recorded while participants attended to their heartbeats. The R-wave of the ECG served as a trigger for EEG averaging. In the latency range of $250-350 \mathrm{~ms}$ after the ECG R-wave, the HEP amplitude at the right central location was significantly higher in good heartbeat perceivers. A significantly positive correlation was observed between the heartbeat perception score and the mean HEP amplitude. Our results confirm that the accuracy of heartbeat perception is reflected in the amplitude of the HEP. Thus, the HEP may be a suitable research tool for the study of brain processes related to visceral perception.
\end{abstract}

Descriptors: Event-related potential, Interoception, Heartbeat perception, Heartbeat-evoked potential

Mental processes related to visceral activity have gained growing interest during the last few years. Damasio's somatic marker hypothesis (Bechara, Damasio, \& Damasio, 2000; Damasio, $1994,2000)$ is an example of a modern psychological theory incorporating the feedback from the periphery (somatosensory and visceral) to the cortex. Its key idea is that many mental processes are influenced by "marker" signals arising through bioregulatory processes. Considering emotions, which to a great extent are expressed through the representation of bodily changes, Damasio (2000, p. 287) speaks of the "obligate bodyrelatedness of feelings." However, up to now, higher brain processes related to the representation of visceroafferent signals have still been poorly understood.

Knowledge about the cortical projection and functional organization of visceral sensations in humans, compared to somatosensation, is sparse. The viscera are innervated by vagal and spinal sensory fibers: The vagus, as a mixed nerve, carries visceral afferents and efferents. The majority (80-85\%) of vagal fibers are afferent, projecting viscerotopically to the nucleus of the solitary tract (NTS) of the brain stem (Jaening, 1995, 1996). Another part of the NTS receives input from the spinal tract. The NTS projects to the parabrachial nuclei as a pontine relay station and the locus coeruleus. Projections then go to multiple higher centers such as the hypothalamus and the thalamus before the cortex is reached. Here, four regions are candidates for the

Address reprint requests to: Rainer Schandry, Biological Psychology, Department of Psychology, Ludwig-Maximilians-University of Munich, Leopoldstr. 13, D-80892, Munich, Germany. E-mail: schandry@psy. uni-muenchen.de. processing of visceroafferent signals: somatosensory cortex, cingulated gyrus, frontal cortex, and insular cortex (cf. Cameron, 2001). The evidence pertaining to the latter stems mainly from animal research, as well as from a few brain-imaging and eventrelated potential (ERP) studies.

As compared to brain-imaging techniques like fMRI, viscerally evoked brain potentials have some advantages. (1) They allow the investigation of cortical processes on a much finer timescale as compared to imaging studies. This is of importance if brain processes are to be observed in response to short pulsate signals. (2) The restrictions induced by the methodology are much less (no immobility of the subject, more possibilities for stimulus presentations, less ambient noise). (3) Finally, the results, in contrast to those found with fMRI, depend only to a comparably small degree on the type of "standard" condition that is chosen as a reference for the experimental condition.

Most ERP studies investigating the cortical representations of visceral sensations have used signals arising from the alimentarygastrointestinal tract or cardiovascular system. Stimulation of the esophagus resulted primarily in evoked potentials generated in the secondary somatosensory cortex (Aziz et al., 1995; Aziz, Schnitzler, \& Enck, 2000; Furlong et al., 1998; Schnitzler et al., 1999). Additionally, visceral sensations following esophageal stimulation appear to be represented in paralimbic and limbic structures, as well as in the insular, anterior cingulated, and prefrontal cortices (Aziz et al., 1995, 2000; Furlong et al., 1998).

The cortical processing of signals from the cardiovascular system by using a heartbeat-evoked potential (HEP) was first addressed by Schandry, Sparrer, and Weitkunat (1986) and by Jones, Leonberger, Rouse, Caldwell, and Jones (1986). 
Subsequent studies provided further evidence for the existence of a HEP (Dirlich, Dietl, Vogl, \& Strian, 1998; Dirlich, Vogl, Plaschke, \& Strian, 1997; Leopold \& Schandry, 2001; Montoya, Schandry, \& Müller, 1993; Riordan, Squires, \& Brener, 1990; Schandry \& Montoya, 1996). The HEP has been reported to appear mainly over two brain regions, the somatosensory cortex and the frontal/prefrontal cortex. Montoya et al. (1993), Leopold and Schandry (2001), and Dirlich et al. (1998) used multichannel recording and observed an HEP appearing as a positive potential shift about $250-600 \mathrm{~ms}$ after the R-wave. Montoya et al. and Leopold and Schandry reported HEP activity primarily at frontal electrodes, whereas Dirlich et al. observed a more posterior distribution of the HEP. Frontal HEP activity is in accordance with knowledge from neuroanatomy (Nieuwenhuys, Voogd, \& Van Huijzen, 1988). Hanamori, Kunitake, Kato, and Kannan (1998) have demonstrated that neurons in the insular cortex of rats were activated when baroreceptors and chemoreceptors were stimulated and emphasized the importance of the insular cortex for the regulation of cardiovascular activity. The insular cortex, the fifth lobe of the brain, is buried in the lateral sulcus and covered by the opercular parts of the frontal, parietal, and temporal lobes. The anterior insula-belonging to the paralimbic system - is believed to contribute to affective and cognitive responses to pain (Aziz et al., 2000).

A small number of imaging studies investigated the cerebral correlates of cardiovascular activity. Critchley, Corfield, Chandler, Mathias, and Dolan (2000) demonstrated an increased regional cerebral blood flow $(\mathrm{rCBF})$ in the right anterior cingulated gyrus and the right anterior insular cortex, as well as a decreased $\mathrm{rCBF}$ in prefrontal and medial temporal regions during physical exercise and mental stressor tasks. King, Menon, Hachinski, and Cechetto (1999) showed increased neuronal activity in response to cardiopulmonary stimuli in the insular cortex as well as in the posterior regions of the thalamus and in the medial prefrontal cortex. In studies with patients suffering from silent ischaemia and angina pectoris (Rosen et al., 1994, 1996), activation in the thalamus and the prefrontal and the cingulated cortex was reported.

Apart from studies considering the cerebral and, especially, cortical representation of heart activity, a second line of research is concerned with the processing of cardio-afferent signals: interoception of cardiac activity ("cardiac awareness," "heartbeat perception"). Heartbeat perception has been studied under many different conditions. One common observation is that there are substantial interindividual differences in heartbeat perception. The ability to perceive cardiac activity seems to depend on such factors as gender, percentage of body fat, and physical fitness (Cameron, 2001; Jones, 1994). Significant differences in heartbeat perception ability were also observed in different clinical samples: Mussgay, Klinkenberg, and Rüddel (1999) demonstrated a tendency toward lower perception scores in patients with depressive, somatoform, and personality disorders, whereas Ehlers, Margraf, and Roth (1988) and Ehlers, Mayou, Sprigins, and Birkhead (2000) reported more accurate heartbeat perception in panic patients. Patients suffering from arrhythmias and benign palpitations (Ehlers et al., 2000) and patients with diabetic neuropathy (Leopold \& Schandry, 2001) showed a decreased heartbeat perception ability compared to healthy controls.

The relation between heartbeat perception and the heartbeatevoked potential has been investigated in a few studies, yielding inconsistent results. Montoya et al. (1993) found a reduced frontotemporal positivity in poor perceivers as compared to good perceivers only when comparing an attention condition with a distraction condition. Schandry et al. (1986) observed group differences between good and poor heartbeat perceivers when submitting the evoked potentials to principle component analysis. Katkin, Cestaro, and Weitkunat (1991) found a significant relationship between a heartbeat-evoked potential (they call it an "N1") recorded in the right hemisphere and the heartbeat detection accuracy. In these studies, only small sample sizes and/or only a few electrodes were used. Thus, it remains an open question whether a brain electrical correlate of the processing of cardio-afferent information can be identified.

The primary hypothesis of the present study was concerned with whether a relationship between heartbeat perception - as a trait variable - and the amplitude of the HEP exists. Additionally, we wanted to provide further evidence to answer the question concerning which brain regions are involved in the processing of cardio-afferent signals.

\section{Method}

\section{Participants}

Forty-four students ( 32 female, 12 male) between 19 and 40 years of age (mean 25.7 years, $S D$ 5.2) participated in the experiment, receiving $20 €$ (about 18 USD) for their participation. They were mainly recruited from an introductory psychology course at the University of Munich. All participants were healthy with no history of heart disease.

\section{Experimental Procedure}

Upon arrival, participants were given written information about the experiment, their informed consent was obtained, and they filled in a general questionnaire concerning personal data (age, educational level, etc.). The participants were then seated in a sound-attenuated chamber connected to the adjacent equipment room by intercom. After attachment of the EEG electrode cap and EOG and ECG electrodes, a first (of two) heartbeat perception task was performed, consisting of four heartbeatcounting phases $(150,120,130$, and 100 cardiac cycles, identified by ECG). During these trials, participants were asked to count their own heartbeats silently. The beginning and end of the counting phases were signaled by a start and stop tone. During heartbeat counting, participants should not take their pulse or attempt to use other manipulations facilitating the counting of heartbeats. They were instructed that in cases where sensory perception of heart activity was lacking, they should nevertheless try to count in a rhythm similar to their assumed heart rhythm. After the stop signal, participants were required to verbally report the number of counted heartbeats, and then the next counting phase started. Participants were not informed about the length of the counting phases nor about their performance. After the first four phases, a break was introduced to let the participants recover from the heartbeat perception task. Thereafter, a silent movie clip (The Gold Rush by Charlie Chaplin) lasting about $15 \mathrm{~min}$ was shown. Subsequently, the last four heartbeat-counting tasks (150, 100, 140, and 110 cardiac cycles) were performed in the above-described manner.

\section{Psychophysiological and Behavioral Data Assessment}

Nonpolarizable silver-silver chloride electrodes were used. Electrode impedance was kept below $8 \mathrm{k} \Omega$. 
EEG activity was recorded from 27 leads. They were attached by use of an electrode cap ("easy cap"; Falk Minow Services) at the following positions: FP1, FP2, F3, F4, F7, F8, FZ, FC1, FC2, FC5, FC6, CZ, C3, C4, CP1, CP2, CP5, CP6, PZ, P3, P4, $\mathrm{P} 7, \mathrm{P} 8, \mathrm{~T} 7, \mathrm{~T} 8, \mathrm{O} 1$, and $\mathrm{O} 2$. Linked mastoids served as a reference. Horizontal EOG was recorded with two electrodes placed lateral to the outer canthus of each eye; vertical EOG was picked up with electrodes above and below the left eye. Signals were amplified (bandpass: $0.1-200 \mathrm{~Hz}$; SYNAMPS, Neuroscan) and digitized at a sampling rate of $250 \mathrm{~Hz}$. Data were bandpass filtered $(0.1-30 \mathrm{~Hz})$ off-line.

ECG electrodes were placed on the left and the right wrist. ECG activity was recorded in the same manner as the EEG with regard to sampling rate $(250 \mathrm{~Hz})$ and filtering. The upstrokes of the ECG R-waves were detected online electronically and were stored as triggers ("R-wave-triggers") on a separate channel to be used for later off-line EEG averaging.

\section{Data Analysis}

Analysis of ERP data. EEG was examined for ocular, myoelectric, and other sources of electrophysiological artefacts. The analysis software (Brain Vision) allowed EOG correction for blinks based on the blink correction method suggested by Gratton, Coles, and Donchin (1983). Epochs were rejected from the analysis if the scalp EEG in any channel exceeded $\pm 80 \mu \mathrm{V}$. These epochs (approximately 16\% of the trials) were eliminated prior to averaging.

EEG epochs were computed extending from $200 \mathrm{~ms}$ prior to and up to $824 \mathrm{~ms}$ after the R-wave-trigger.

Data were corrected for cardiac field artefacts by using the Hjorth source derivation method (Hjorth, 1975). This method yields an estimation of the source activity as it appears at the scalp surface for each individual electrode. It is realized in the 1020 system of electrode placement basically as an analogue superposition of four bipolar derivations, forming a starlike configuration around each electrode. The weighted activity of the surrounding electrodes is subtracted from each electrode; thus, cardiac field effects should be removed to a great extent in the remaining EEG activity. Montoya (1993) described this method as being sufficient to eliminate the cardiac field.

Statistical analysis of ERP and ECG data. In accordance with former results indicating highest HEP amplitudes in the latency range of 250-350 ms (Leopold \& Schandry, 2001; Schandry et al., 1986), analyses were performed on the mean voltage within this time window. Between-group differences were investigated by submitting the data to ANOVAs with seven levels of Aggregated Scalp Sectors (frontal: F3, F4, F7, F8, FP1, FP2; frontocentral: FC1, FC2, FC5, FC6; central: C3, C4; centroparietal: CP1, CP2, CP5, CP6; parietal: P3, P4, P7, P8; temporal: T7, T8; occipital: O1, O2), two levels of Hemisphere (left/right), and two levels of Heartbeat Perception (good/poor heartbeat perceivers). Main effects for the variables Aggregated Scalp Sectors, Hemisphere, and Heartbeat Perception, as well as interaction effects between (1) Hemisphere and Heartbeat Perception, and (2) Aggregated Scalp Sectors and Hemisphere and Heartbeat Perception were investigated. The respective results are only reported when significant effects were obtained.

For all the results reported, the Huynh-Feldt correction was applied when sphericity assumptions were violated. In these cases corrected probability levels are reported.
The relation between heartbeat perception and the HEP was examined with bivariate nonparametric correlation analyses (Spearman rho) between the heartbeat perception score and the mean HEP amplitude in the latency range of 250-350 ms.

ECG amplitudes were calculated as the mean of all data points in the time window of $250-350 \mathrm{~ms}$. Between-group differences in ECG amplitudes were investigated by submitting the data to an univariate ANOVA with the factor Heartbeat Perception (good/poor heartbeat perceivers).

Analysis of heartbeat perception. A heartbeat perception score was calculated according to the following equation:

$$
\begin{aligned}
\text { Perception score }= & 1 / 8 \sum(1-(\mid \text { recorded heartbeats }- \\
& \text { counted heartbeats } \mid) / \text { recorded heartbeats })
\end{aligned}
$$

High scores (maximum 1) indicate absolute accurate heartbeat perception ability. A cutoff score of .85 was used to categorize subjects either as good or poor heartbeat perceivers. This is in accordance with the cutoff selected by Montoya et al. (1993) and Weitkunat and Schandry (1995).

\section{Results}

\section{Heartbeat Perception}

The mean heartbeat perception score was .79 (SD .14, minimum .42 , maximum .98). No significant differences in the heartbeat perception scores occurred between men and women (univariate ANOVA: $F(1,42)=1.03, p=$ n.s. $)$.

A total of 18 subjects (14 female, 4 male) were identified as good heartbeat perceivers (cutoff score: .85, mean .92, $S D .04$, minimum $=.88$, maximum $=.98)$. Twenty-six subjects $(18$ female, 8 male) were classified as poor heartbeat perceivers (mean .71, $S D .12$, minimum .42, maximum .84).

\section{HEP Morphology and Scalp Distribution}

Grand averages of all electrode positions for good and poor heartbeat perceivers are depicted in Figure 1. At most electrodes, a relatively broad waveform was registered in the latency range of $250-500 \mathrm{~ms}$. The polarity of the peak of this waveform varies across electrode locations. However, in the latency range from 250 to $350 \mathrm{~ms}$, which has repeatedly been shown (Leopold \& Schandry, 2001; Schandry et al., 1986) to be closely related to heart activity, the picture becomes more unequivocal. The distribution of the mean HEP amplitude for the latency range of $250-350 \mathrm{~ms}$ is depicted in Figure 2. The ANOVA performed for the latency range of $250-350 \mathrm{~ms}$ revealed a significant main effect for the factor Aggregated Scalp Sectors, $F(6,252)=24.44$, $\left.p<.001, \eta^{2}=.37, \varepsilon=1.00\right)$. The HEP was most pronounced over central and frontocentral electrode positions, appearing as a positive mean activity in this latency range.

\section{Differences in the HEP between Good and Poor Heartbeat Perceivers}

In Figure 3, the scalp distribution of the group means of the HEP amplitude of good versus poor heartbeat perceivers is depicted for the latency range of $250-350 \mathrm{~ms}$. As a main effect, the mean HEP activity of good heartbeat perceivers was significantly larger than that of poor heartbeat perceivers (mean HEP activity of $0.06 \mu \mathrm{V}$ as compared to $-0.04 \mu \mathrm{V}, F(1,42)=5.89, p<.05$, $\eta^{2}=.12, \varepsilon=.66$ ). A significant interaction effect between the factors of Aggregated Scalp Sectors, Hemisphere, and Heartbeat Perception, $F(6,252)=3.09, p<.05, \eta^{2}=.07, \varepsilon=.86$, indicated 


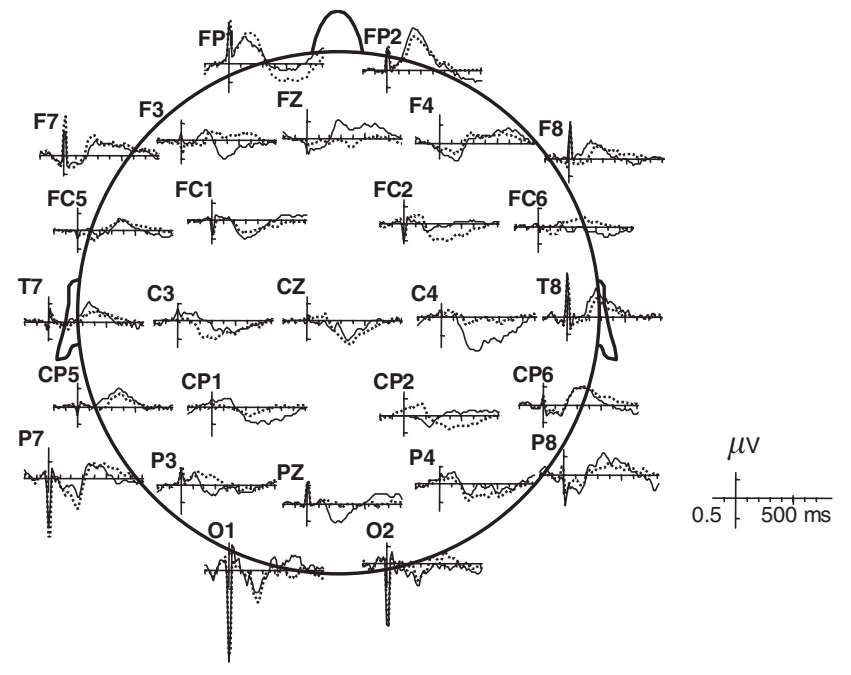

Figure 1. HEP morphology contrasting good (black line) and poor (dashed line) heartbeat perceivers.

significant differences between good and poor heartbeat perceivers at specific scalp areas. Separate two-way analyses of variance (ANOVA), conducted for all Aggregated Scalp Sectors, showed that the HEP amplitude for good heartbeat perceivers $(N=18$, mean $0.73 \mu \mathrm{V}, S D=0.84)$ was significantly larger at the right central electrode site than for poor heartbeat perceivers $(N=26$, mean $0.15 \mu \mathrm{V} ; S D=0.52 ; F(1,42)=8.30, p<.01$, $\left.\eta^{2}=.17, \varepsilon=.80\right)$.
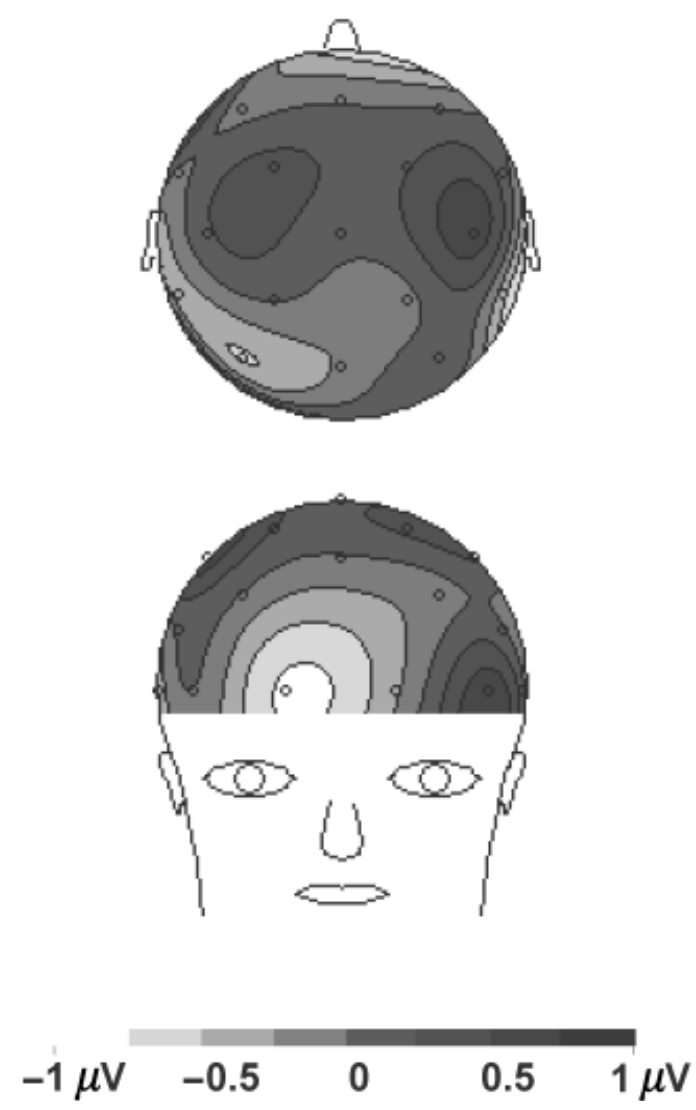

Figure 2. Mean HEP activity in the latency range of $250-350 \mathrm{~ms}$.

\section{ECG Analysis}

ECG mean scores did not differ significantly between good and poor heartbeat perceivers in the latency range of $250-350 \mathrm{~ms}$, $F(1,42)=.01, p=$ n.s.

\section{Correlation Analyses}

Taking into account the observed maximum HEP activity over the right central electrode position (i.e., at $\mathrm{C} 4$ ), we calculated the correlation between the mean HEP amplitude at C4 and the heartbeat perception score. This resulted in a significantly positive correlation of $r=.28(N=44, p<.05)$.

\section{Discussion}

A heartbeat-evoked potential was observed as a broad waveform in the latency range later than $200 \mathrm{~ms}$ post-R-wave. The mean HEP activity was higher in the group of good heartbeat perceivers as compared to poor heartbeat perceivers. Moreover, a significantly positive correlation was observed between the mean HEP amplitude in the time window of 250-350 ms and the accuracy of heartbeat perception.

Concerning the observed frontocentral scalp distribution of the HEP, our data is in accordance with the work by Montoya et al. (1993), Riordan et al. (1990), Schandry et al. (1986) and Schandry and Montoya (1996). Dirlich et al. (1997, 1998), however, reported a HEP activity over the parietal cortices.

Following the reasoning of Aziz et al. (1995), for instance, this frontocentral activity pattern may reflect sources in the insular cortex and/or the somatosensory cortices. In our opinion, the insular cortex is a most probable source of brain activity being triggered by cardio-afferent signals. It is a well-established

\section{Good heartbeat perceivers}

\section{Poor heartbeat perceivers}
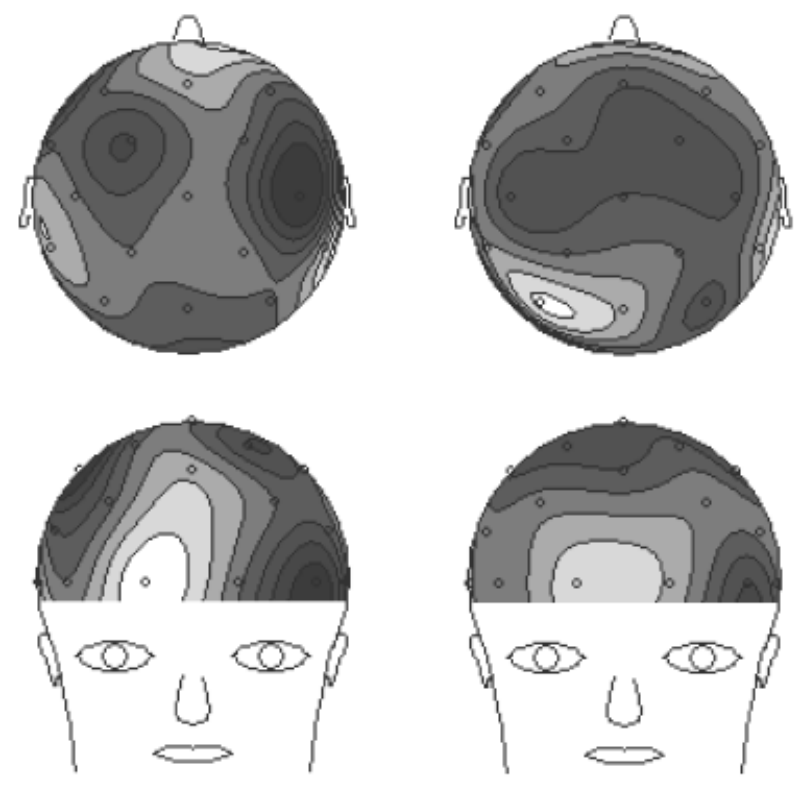

$$
-1 \mu \mathrm{V}-0.5
$$

\section{0}

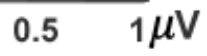

Figure 3. Mean HEP activity for good and poor heartbeat perceivers in the latency range of $250-350 \mathrm{~ms}$. 
finding that this region plays an important role for the regulation of cardiovascular activity (Augustine, 1996; Bennarroch, 1993; Hanamori et al., 1998; Oppenheimer, Saleh, \& Cechetto, 1992). Using functional imaging, it could be demonstrated that increased neuronal activity in the insular cortex is associated with cardiovascular arousal (Critchley et al., 2000; King et al., 1999). The importance of the insular cortex for processing visceroafferent signals is underlined by studies of esophagealevoked potentials (Aziz et al., 1995, 2000; Franssen, Weusten, Wineneke, \& Smout, 1996; Furlong et al., 1998; Schnitzler et al., 1999). Aziz et al. showed that the early components (N100) evoked by stimulation of the distal esophagus were distributed predominantly over central areas (C3 and C4), whereas longer latency components (N200, P200) showed maximal distribution over frontal (F3, F4, FZ) and central locations. As probable sources for esophageal-evoked potentials, Franssen et al. reported dipoles in the cingulated gyri and the insular cortex. Within this context, it should not be overlooked that there is growing evidence that the autonomic and sensory innervations of the esophagus and of the heart are closely related (Bajwa et al., 1997; Fallen, Kamath, Tougas, \& Upton, 2001; Hollerbach et al., 1997, 2001; Tougas et al., 1997).

In the present study, HEP activity was seen to be strongest over the right hemisphere. This observation is in accordance with former studies (Leopold \& Schandry, 2001; Riordan et al., 1990; Weitkunat, Cestaro, \& Katkin, 1989) demonstrating higher HEP amplitudes over the right than the left hemisphere. From the work of Hari et al. (1993) it is known that the insular cortex as well as the secondary somatosensory areas reveal a higher activity on the side contralateral to the stimulation. Within this context, one has to consider that the heart is not symmetrically oriented relative to the vertical body axes, but points with the apex of the left ventricle to the lower left side. As a consequence of this, the transfer of mechanical energy from the heart to the mechanosensitive tissue of the thorax is more pronounced on the left side of the thorax and triggers stronger somatosensory signals from here as compared to the right side. Due to a predominantly contralateral projection of the afferent fibers from the thorax, the cortical representation of cardiodynamic activity should be more pronounced in the right hemisphere. The importance of the right hemisphere for heart-related brain processes is further supported by data from Yoon, Morillio, Cechetto, and Hachinski (1997) and Ahern et al. (2001). Yoon et al. showed that a pharmacologically induced reduction in heart rate variability and an increase in heart rate was observed only when the substance was applied in the right hemisphere. Ahern et al. demonstrated that the right hemisphere plays a greater role in cerebral regulation of cardiac function when investigating changes in heart rate and heart rate variability in 73 subjects.

The result of higher HEP amplitudes in good heartbeat perceivers than in poor heartbeat perceivers is consistent with former studies. Weitkunat and Schandry (1990) demonstrated an enhancement of the HEP amplitude following cardiac awareness training. They observed the most pronounced effects on the HEP amplitude at frontal electrodes in the time window of 250 $400 \mathrm{~ms}$. Montoya et al. (1993) found an interaction between the factors of Group (good/poor heartbeat perception) and Attention (attention directed to the heart activity vs. distraction): The two groups differed in the HEP amplitude (higher amplitude in good perceivers), but this effect was only significant in the distraction condition. Katkin et al. (1991) demonstrated a significant relationship between the amplitude of an "N1" (not further described in their publication), evoked by the heartbeat recorded at F8 and the heartbeat detection accuracy. As Katkin et al. used a different methodology for measuring cardiac awareness and rejection of cardiac artefacts, their results are not directly comparable to ours.

Further evidence for a direct relationship between visceral awareness and viscerally evoked brain activity merges from recent studies with esophageal evoked potentials. Electrical stimulation of the esophagus with stimuli of varying intensity showed that an increase in sensory perception was associated with a reduction in the latency and an increase in amplitude of the evoked potential components (Hobson et al., 1998; Hobson, Sarkar, Furlong, \& Thompson, 2000). Hollerbach et al. (2001) investigated the effects of cognitive factors involved in visceral perception using esophageal-evoked potentials. Their data indicate that the event-related potential (P300) observed was associated with the attention to and awareness of the actual stimulus. Other studies (Knafelc \& Davenport, 1997; Webster \& Colrain, 2000) with the respiratory-related-evoked potential demonstrated that the perceived magnitude of the respiratory load was related to the amplitude of the P100 and P300 components of the evoked response. Bloch-Salisbury, Harver, and Squires (1998) showed that perceptual sensitivity to inspiratory, flow-resistive loads was significantly correlated with the amplitude of the parietal P300 response, reporting correlation coefficients of up to .57 for stimuli at a near-threshold level.

Congruent results pertaining to a reflection of perceptual accuracy in the evoked potential are derived from studies using visual and auditory stimuli (Devrim, Demiralp, Kurt, \& Yükcesir, 1999; Hillyard, Squires, Bauer, \& Lindsay, 1971; Picton, Hillyard, Krausz, \& Galambos, 1974; Trejo, Kramer, \& Arnold, 1995). Trejo et al., for example, found that the detection of visual stimuli at sensory threshold is positively related to the P300 amplitude.

Other studies using external, near-threshold, somatosensory stimuli have shown that evoked potential amplitudes following detected stimuli are higher than those following missed stimuli. Kekoni, Hämäläinen, McCloud, Reinikainen, and Näätänien (1996) used vibration stimuli and showed that the N250 of the SEP was positively related to the detection of target stimuli. Hashimoto, Yoshikawa, and Kimura (2000) demonstrated that the late P300 of the somatosensory-evoked potentials could correctly predict the perception of a threshold air stimulus: A P300 late component occurred only in response to detected stimuli and was not found in response to undetected stimuli.

We observed a significantly positive correlation between the HEP amplitude and the heartbeat perception score. It may be assumed that the common factor influencing the HEP amplitude as well as the heartbeat perception score is the intensity of the eliciting stimulus. A strong relation between cardiodynamic parameters and heartbeat perception has been shown by Schandry, Bestler, and Montoya (1993) and Schandry and Bestler (1995). They reported significant correlations (up to .83) between the heartbeat perception score and different cardiodynamic parameters, that is, stroke volume, blood ejection velocity, momentum of the ejected blood mass, and contractility. For external stimuli (visual, auditory, somatosensory), it is a wellestablished fact that the stimulus intensity influences the amplitudes of the evoked potentials (e.g., Carrillo-de-la-Pena, Rodriguez Holguin, Corral, \& Cadaveira, 1999; Cass \& Polich, 1997; Covington \& Polich, 1996; Nakajima \& Imamura, 2000; Shimojo, Svensson, Arendt-Nielsen, \& Chen, 2000). Also recent 
studies with esophageal-evoked potentials showed that an increase of stimulus intensity was associated with an increase in the amplitude of the esophageal-evoked potential components (Fallen et al., 2001; Hobson et al., 1998, 2000).
As a general conclusion, we state that the HEP is a valid reflection of brain processes related to cardiac perception. It may serve as a model phenomenon for further research in the area of viscerocortical interactions.

\section{REFERENCES}

Ahern, G. L., Sollers, J. J., Lane, R. D., Labiner, D. M., Herring, A. M., Weinand, M. E., Hutzler, R., \& Thayer, J. F. (2001). Heart rate and heart rate variability changes in the intracarotid sodium amobarbital test. Epilepsia, 42, 912-921.

Augustine, J. R. (1996). Circuitry and functional aspects of the insular lobe in primates including humans. Brain Research Reviews, 22, 229-244.

Aziz, Q., Furlong, P. L., Barlow, J., Hobson, A., Alani, S., Bancewicz, J., Ribbands, M., Harding, G. F., \& Thompson, D. G. (1995). Topographic mapping of cortical potentials evoked by distension of the human proximal and distal esophagus. Electroencephalography and Clinical Neurophysiology, 96, 219-228.

Aziz, Q., Schnitzler, A., \& Enck, P. (2000). Functional neuroimaging of visceral sensation. Journal of Clinical Neurophysiology, 17, 604-612.

Bajwa, A., Hollerbach, S., Kamath, V., Upton, A. R. M., Fitzpatrick, D., Fallen, E. L., \& Tougas, G. (1997). Neurocardiac response to esophageal electric stimulation in humans: Effects of varying stimulation frequencies. American Journal of Physiology, 272, 896-901.

Bechara, A., Damasio, H., \& Damasio, A. R. (2000). Emotion, decision making and the orbitofrontal cortex. Cerebral Cortex, 10, 295-307.

Bennarroch, E. E. (1993). The central autonomic network: Functional organization, dysfunction, and perspective (Review). Mayo Clinic Proceedings, 68, 998-1001.

Berthoud, H.-R., \& Neuhuber, W. L. (2000). Functional and chemical neuroanatomy of the afferent vagal system. Autonomic Neuroscience: Basic and Clinical, 85, 1-17.

Bloch-Salisbury, E., Harver, A., \& Squires, N. K. (1998). Event-related potentials to inspiratory flow-resistive loads in young adults: Stimulus magnitude effects. Biological Psychology, 49, 165-186.

Cameron, O. G. (2001). Interoception: The inside story-A model for psychosomatic processes. Psychosomatic Medicine, 63, 697-710.

Carrillo-de-la-Pena, M., Rodriguez Holguin, S., Corral, M., \& Cadaveira, F. (1999). The effects of stimulus intensity and age on visual-evoked potentials (VEPs) in normal children. Psychophysiology, 36, 693-698.

Cass, M., \& Polich, J. (1997). P300 from a single-stimulus paradigm: Auditory intensity and tone frequency effects. Biological Psychology, $45,51-65$.

Covington, J. W., \& Polich, J. (1996). P300, stimulus intensity, and modality. Electroencephalography and Clinical Neurophysiology, 100, 579-584.

Critchley, H. D., Corfield, D. R., Chandler, M. P., Mathias, C. J., \& Dolan, R. J. (2000). Cerebral correlates of autonomic cardiovascular arousal: A functional neuroimaging investigation in humans. Journal of Physiology, 523, 259-270.

Damasio, A. R. (1994). Descartes' error: Emotion, reason and the human brain. New York: Grosset/Putman.

Damasio, A. R. (2000). The feeling of what happens: Body, emotion and the making of consciousness. London: Vintage.

Devrim, M., Demiralp, T., Kurt, A., \& Yükcesir, I. (1999). Slow cortical potential shifts modulate the sensory threshold in human visual system. Neuroscience Letters, 270, 17-20.

Dirlich, G., Dietl, T., Vogl, L., \& Strian, F. (1998). Topography and morphology of heart action-related EEG potentials. Electroencephalography and Clinical Neurophysiology, 108, 299-305.

Dirlich, G., Vogl, L., Plaschke, M., \& Strian, F. (1997). Cardiac field effects on the EEG. Electroencephalography and Clinical Neurophysiology, 102, 307-315.

Ehlers, A., Margraf, J., \& Roth, W. T. (1988). Selective information processing, interoception and panic attacks. In I. Hand \& H. U. Wittchen (Eds.), Panic and phobias (Vol. 2, pp. 129-148). Berlin: Springer.

Ehlers, A., Mayou, R., Sprigins, D. C., \& Birkhead, J. (2000). Psychological and perceptual factors associated with arrhythmias and benign palpitations. Psychosomatic Medicine, 62, 693-702.
Fallen, E. L., Kamath, M. V., Tougas, G., \& Upton, A. (2001). Afferent vagal modulation: Clinical studies of visceral sensory input. Autonomic Neuroscience: Basis and Clinical, 90, 35-40.

Ferguson, M. L., \& Katkin, E. S. (1996). Visceral perception, anhedonia, and emotion. Biological Psychology, 42, 131-145.

Franssen, H., Weusten, B., Wineneke, G. H., \& Smout, A. (1996). Source modeling of esophageal evoked potentials. Electroencephalography and Clinical Neurophysiology, 100, 85-95.

Furlong, P. L., Aziz, Q., Singh, K. D., Thompson, D. G., Hobson, A., \& Harding, G. F. A. (1998). Cortical localization of magnetic fields evoked by esophageal distension. Electroencephalography and Clinical Neurophysiology, 108, 234-243.

Gratton, G., Coles, M. G. H., \& Donchin, E. (1983). A new method for off-line removal of ocular artifact. Electroencephalography and Clinical Neurophysiology, 55, 468-484.

Hanamori, T., Kunitake, T., Kato, K., \& Kannan, H. (1998). Responses of neurons in the insular cortex to gustatory, visceral, and nociceptive stimuli in rats. The Journal of Neurophysiology, 79, 2535-2545.

Hari, R., Karhu, J., Hämäläinen, H., Knuutila, J., Salonen, O., \& Sams, M. (1993). Functional organization of the human first and secondary somatosensory cortices: A neuromagnetic study. European Journal of Neuroscience, 5, 724-734.

Hashimoto, I., Yoshikawa, K., \& Kimura, T. (2000). Somatosensory evoked potentials to a threshold air-puff can predict stimulus detection in human subjects. Neuroscience Letters, 282, 181-184.

Hillyard, S. A., Squires, K. C., Bauer, J. W., \& Lindsay, P. H. (1971). Evoked potential correlates of auditory signal detection. Science, 172, $1357-1360$.

Hjorth, B. (1975). An on-line transformation of EEG scalp potentials into orthogonal source derivations. Electroencephalography and Clinical Neurophysiology, 39, 526-530.

Hobson, A. R., Aziz, Q., Furlong, P. L., Barlow, J. D., Bancewicz, J., \& Thompson, D. G. (1998). Identification of the optimal parameters for recording cortical evoked potentials to human esophageal electrical stimulation. Neurogastroenterology and Motility, 10, 421-430.

Hobson, A. R., Sarkar, S., Furlong, P. L., \& Thompson, D. G. (2000). Identification of the optimal parameters for recording cortical evoked potentials by mechanical stimulation of the human esophagus. Neurogastroenterology and Motility, 12, 163-171.

Hollerbach, S., Kamath, M. V., Fitzpatrick, D., Shine, G., Fallen, E., Upton, A. R., \& Tougas, G. (1997). The cerebral response to electrical stimuli in the esophagus is altered by increasing stimulus frequencies. Neurogastroenterology and Motility, 9, 129-139.

Hollerbach, S., May, A., Kamath, M. V., Shine, G., Upton, A. R. M., \& Tougas, G. (2001). Objective assessment of cognitive factors involved in visceral perception by using event-related cerebral evoked responses to esophageal target stimulation in man. Digestive Diseases and Sciences, 46, 790-801.

Jaening, W. (1995). Visceral afferent neurons: Neuroanatomy and functions, organ regulations and sensations. In D. Vaitl \& R. Schandry (Eds.), From the heart to the brain (pp. 5-34). Frankfurt am Main: Verlag Peter Lang, Europäischer Verlag der Wissenschaften.

Jaening, W. (1996). Neurobiology of visceral afferent neurons: Neuroanatomy, functions, regulations and sensations. Biological Psychology, 42, 29-51.

Jones, G. E. (1994). Perception of visceral sensations: A review of recent findings, methodologies, and future directions. In J. R. Jennings, P. K. Ackles, \& M. G. H. Coles (eds.). Advances in psychophysiology (Vol. 5, pp. 55-192). London: Jessica Kingsley Publishers.

Jones, G. E., Leonberger, T. F., Rouse, C. H., Caldwell, J. A., \& Jones, K. R. (1986). Preliminary data exploring the presence of an evoked potential associated with cardiac visceral activity [Abstract]. Psychophysiology, 23, 445.

Katkin, E., Cestaro, V. L., \& Weitkunat, R. (1991). Individual differences in cortical evoked potentials as a function of heartbeat detection ability. International Journal of Neuroscience, 61, 269-276. 
Kekoni, J., Hämäläinen, H., McCloud, V., Reinikainen, K., \& Näätänien, R. (1996). Is the somatosensory N250 related to deviance discrimination or conscious target detection? Electroencephalography and Clinical Neurophysiology, 100, 112-125.

King, A. B., Menon, R.S, Hachinski, V., \& Cechetto, D. F. (1999). Human forebrain activation by visceral stimuli. The Journal of Comparative Neurology, 413, 572-582.

Knafelc, M., \& Davenport, P. W. (1997). Relationship between resistive loads and $\mathrm{P}_{1}$ peak of respiratory-related evoked potential. Journal of Applied Physiology, 83, 918-926.

Leopold, C., \& Schandry, R. (2001). The heartbeat-evoked brain potential in patients suffering from diabetic neuropathy and in healthy control persons. Clinical Neurophysiology, 112, 674-682.

Montoya, P. (1993). Herzwahrnehmung und hirnelektrische Aktivität. Frankfurt am Main: Peter Lang-Verlag.

Montoya, P., Schandry, R., \& Müller, A. (1993). Heart-beat evoked potentials (HEP): Topography and influence of cardiac awareness and focus of attention. Electroencephalography and Clinical Neurophysiology, 88, 163-172.

Mussgay, L., Klinkenberg, N., \& Rüddel, H. (1999). Heart beat perception in patients with depressive, somatoform, and personality disorders. Journal of Psychophysiology, 13, 27-36.

Nakajima, Y., \& Imamura, N. (2000). Relationship between attention effects and intensity effects on the cognitive N140 and P300 components of somatosensory ERPs. Clinical Neurophysiology, 111, 1711-1718.

Nieuwenhuys, R., Voogd, J., \& Van Huijzen, C. (1988). The human central nervous system - $A$ synopsis and atlas (3rd ed). New York: Springer.

Oppenheimer, S. M., Saleh, T., \& Cechetto, D. F. (1992). Lateral hypothalamic area neurotransmission and neuromodulation of the specific cardiac effects of insular cortex stimulation. Brain Research, 581, 133-142.

Picton, T. W., Hillyard, S. A., Krausz, H. I., \& Galambos, R. (1974). Human auditory evoked potentials. I. Evaluation of components. Electroencephalography and Clinical Neurophysiology, 87, 235-241.

Riordan, H., Squires, N. K., \& Brener, J. (1990). Cardio-cortical potentials: Electrophysical evidence for visceral perception [abstract]. Psychophysiology, 27, 559.

Rosen, S. D., Paulesu, E., Frith, C. D., Frackowiak, R. S., Davies, G. J., Jones, T., \& Camici, P. G. (1994). Central nervous pathways mediating angina pectoris. The Lancet, 344, 147-150.

Rosen, S. D., Paulesu, E., Nihoyannopoulos, P., Tousulis, D., Frackowiak, R. S. J., Frith, C. D., Jones, T., \& Camici, P. G. (1996). Silent ischemia as a central problem: Regional brain activation compared in silent and painful myocardiac ischemia. Annals of Internal Medicine, 124, 939-949.
Schandry, R., \& Bestler, M. (1995). The association between parameters of cardiovascular function and heartbeat perception. In D. Vaitl \& R. Schandry (Eds.) From the heart to the brain (pp. 105-120). Frankfurt am Main: Verlag Peter Lang, Europäischer Verlag der Wissenschaften.

Schandry, R., Bestler, M., \& Montoya, P. (1993). On the relation between cardiodynamics and heartbeat perception. Psychophysiology, $30,467-474$.

Schandry, R., \& Montoya, P. (1996). Event-related brain potentials and the processing of cardiac activity. Biological Psychology, 42, 75-85.

Schandry, R., Sparrer, B., \& Weitkunat, R. (1986). From the heart to the brain: A study of heartbeat contingent scalp potentials. International Journal of Neuroscience, 22, 261-275.

Schnitzler, A., Volkmann, J., Enck, P., Frieling, T., Witte, O. W., \& Freund, H.-J. (1999). Different cortical organization of visceral and somatic sensation in humans. European Journal of Neuroscience, 11, 305-315.

Schnitzler, A., \& Enck, P. (2000). Functional neuroimaging of visceral sensation. Journal of Clinical Neurophysiology, 17, 604-612.

Shimojo, M., Svensson, P., Arendt-Nielsen, L., \& Chen, A. C. (2000). Dynamic brain topography of somatosensory evoked potentials and equivalent dipoles in response to graded painful skin and muscle stimulation. Brain Topography, 13, 43-58.

Tougas, G., Kamath, M., Watteel, G., Fitzpatrick, D., Fallen, E., Hunt, R. H., \& Upton, A. R. M. (1997). Modulation of neurocardiac function by esophageal stimulation in humans. Clinical Science, 92, 167-174.

Trejo, L. J., Kramer, A. F., \& Arnold, J. A. (1995). Event-related potentials as indices of display-monitoring performance. Biological Psychology, 40, 33-71.

Webster, K., \& Colrain, I. M. (2000). The relationship between respiratory-related evoked potentials and the perception of inspiratory resistive loads. Psychophysiology, 37, 831-841.

Weitkunat, R., Cestaro, V., \& Katkin, E. S. (1989). Evidence for a lateralized heartbeat evoked potential. Psychophysiology, 26(suppl.), 65.

Weitkunat, R., \& Schandry, R. (1990). Motivation and heartbeat evoked potentials. Journal of Psychophysiology, 4, 33-40.

Weitkunat, R., \& Schandry, R. (1995). Cortical evoked potentials and heartbeat perception. In D. Vaitl \& R. Schandry (Eds), From the heart to the brain (pp. 105-120). Frankfurt am Main: Verlag Peter Lang, Europäischer Verlag der Wissenschaften.

Yoon, B. W., Morillio, C. A., Cechetto, D. F., \& Hachinski, V. (1997). Cerebral hemispheric lateralization in cardiac autonomic control. Archives of Neurology, 54, 741-744.

(ReCEIVed July 31, 2002; ACCEPTED March 3, 2003) 